Fecha de recepción: febrero 2019

Fecha de aceptación: julio 2019

Versión final: noviembre 2020

\section{En el laberinto del tiempo. El videojuego y la evolución de la narrativa}

Federico Alvarez Igarzábal *

Resumen: Los videojuegos ofrecen la opción de guardar y cargar partidas, permitiendo a los jugadores retroceder en el tiempo del juego. Cada vez que un evento no deseado tiene lugar, estos pueden cargar una partida previa y jugar una porción del juego nuevamente para obtener el resultado anhelado. Dos desafíos surgen de esta mecánica a la hora de contar historias: 1) La capacidad de deshacer eventos es incompatible con la tragedia y 2) emerge una paradoja temporal: Al volver atrás en el tiempo, el jugador conoce el futuro. El personaje que este controla no puede poseer ese conocimiento porque fue restablecido con el resto del mundo ficcional. Pero, dado que es controlado por el jugador, se comporta como si lo tuviera.

Palabras clave: videojuegos - narrativa - narrativa multilineal - ficción - interacción - tiempo - literatura.

[Resúmenes en inglés y portugués en las páginas 50 - 51]

${ }^{(*)}$ Investigador en el Institute for Frontier Areas of Psychology and Mental Health en Friburgo, Alemania. Realizó sus estudios de doctorado en la Universidad de Colonia y la Universidad de Ciencias Aplicadas de Colonia, Alemania. Su tesis, titulada "Time and Space in Video Games", se basó en el análisis del tiempo en los videojuegos desde una perspectiva formalista y cognitivista.

¿Quién no anheló alguna vez poder volver atrás en el tiempo? ¿Cuántas veces quisiéramos poder rebobinar para evitar acontecimientos vergonzosos, dolorosos, o simplemente inconvenientes? La flecha del tiempo, sin embargo, continúa con rigor hacia adelante. Somos viajeros en el tiempo en una calle con una única dirección.

En la narrativa, la irreversibilidad del tiempo es una fuente rica de tensión dramática, enfatizada quizás con mayor vigor en la tragedia. Los amantes Romeo y Julieta mueren al final de la historia. El rey Edipo descubre que el adivino Tiresias y el oráculo de Apolo estaban en lo cierto: Él mismo mató a su padre y desposó a su madre, con quien luego tuvo hijos. Es el carácter definitivo de los hechos lo que les otorga su potencia dramática. No hay nada que los personajes o la audiencia puedan hacer para evitarlos o revertirlos. Aunque no todo videojuego relata necesariamente una historia, el medio posee un formi- 
dable potencial narrativo. Este potencial radica, entre otras cosas, en el hecho de que la audiencia se convierte en protagonista y puede tomar decisiones que influencian la sucesión de eventos. Gracias al carácter interactivo del videojuego, los jugadores son actores en mundos ficcionales en los que la acción puede tener dos o más desenlaces. Es decir, la acción en un videojuego se desarrolla de un modo multilineal, ${ }^{1}$ a diferencia del cine, la televisión o la literatura, medios en los que las historias siguen típicamente una única línea. ${ }^{2}$ Incluso en juegos con premisas narrativas simples, como Super Mario Bros. (Nintendo, 1985), la acción puede concluir de dos formas distintas: Mario salva a la princesa o muere en el intento-si tenemos en cuenta la cantidad de maneras en que Mario puede morir, el número de finales posibles aumenta radicalmente. Los jugadores, al tener control sobre el protagonista, son responsables de conducirlo al final más favorable. La conexión entre personajes y jugadores es, a diferencia de las narrativas tradicionales, una de responsabilidad compartida. Si el personaje fracasa o triunfa, también lo hace el jugador.

Sumado al control sobre uno o más personajes, es común que los jugadores puedan manipular el tiempo en los videojuegos. Al momento de guardar una partida, los jugadores se aseguran de preservar el progreso realizado hasta ese punto. Llegado el caso de que el personaje muera u ocurra algún otro evento no deseado, los jugadores pueden cargar la partida guardada y volver atrás en el tiempo del juego para intentar de nuevo. Es decir, los videojuegos otorgan esa tan anhelada capacidad de manipular la flecha del tiempo para deshacer acontecimientos negativos.

Esta facultad de retroceder en el tiempo abre fascinantes caminos para el desarrollo del arte narrativo, a la vez que introduce nuevos desafíos y problemas. Pero, antes de adentrarnos en mayor profundidad en estas dificultades, es pertinente echar un breve vistazo al reciente desarrollo de la narración multilineal e interactiva.

\section{Senderos que se Bifurcan}

Las ideas seminales que llevaron a la tecnología digital con la que se desarrollan videojuegos ya se manifiestan en el pensamiento de Vannebar Bush (2003 [1945]) en la década de 1940-principalmente en su influyente artículo As We May Think, en el cual propuso el afamado Memex, una especie de protocomputadora mecánica diseñada para proveer rápido acceso a información almacenada en microfilm. En las décadas subsiguientes, J.C.R. Licklider (2003 [1960]), Douglas Engelbart (2003 [1962]) y Ted Nelson (2003 [1965]), entre muchos otros, hacen eco de las ideas de Bush y comienzan a sentar las bases teóricas y tecnológicas de nuestro presente mundo digital, en un esfuerzo histórico dirigido a potenciar el intelecto humano-en palabras de Engelbart (2003 [1962]). Una de las centrales preocupaciones de esta generación de pioneros era generar sistemas que permitieran el almacenamiento, rápido acceso y efectiva manipulación de grandes volúmenes de información. Entre los conceptos que propusieron con el fin de optimizar la navegación a través de cantidades potencialmente abrumadoras de datos, se encuentran los enlaces y el hipertexto-término acuñado por Nelson (2003 [1965], p. 144). Es en este contexto que se asienta el suelo fértil en que hoy germinan un sinnúmero de narrativas hipertextuales, in- 
teractivas y multilineales-así como las herramientas para concebirlas, como el programa Storyspace o los más recientes motores gráficos (cf. Murray, 2003).

Pero algunos años antes de que Bush publicara sus ideas, un afamado escritor ya estaba imaginando la narrativa multilineal. En El Jardín de Senderos que se Bifurcan (Borges, 2013 [1941], pp.146-157), Jorge Luis Borges describe una novela ficticia homónima del (asimismo ficticio) escritor Ts'ui Pên. Esta novela forma parte de un proyecto que le ocupó al autor chino los últimos trece años de su vida. Sus objetivos eran "escribir una novela que fuera más popular que el Hung Lu Meng” y "edificar un laberinto en el que se perdieran todos los hombres” (ibíd., p.150). Luego de su muerte, Ts'ui Pên solo deja atrás una serie de fragmentos del proyecto literario y ningún rastro de un laberinto. Hacia el final de la historia, Borges revela que el escritor no fracasó en su empresa: El laberinto y la novela son de hecho la misma entidad. La intención de Ts'ui Pên no era crear un laberinto espacial sino temporal, una novela compuesta por fragmentos contradictorios que relaten versiones alternativas de la historia—en palabras de Borges: una "trama de tiempos que se aproximan, se bifurcan, se cortan o que secularmente se ignoran” (ibíd., p. 156).

Como remarca el poeta y profesor del MIT Nick Montfort (2003, p. 30): "Our use of computers is also based on those who, like Borges (...), saw those courses that future artists, scientists, and hackers might take". Las ideas de Borges se anticipan así al estado actual de la ficción interactiva y multilineal que ofrecen los videojuegos. Previo a la narrativa multilineal, se desarrollaría la narrativa hipertextual en el formato tradicional del libro-en obras como Pálido Fuego de Vladimir Nabokov (2003 [1962]), o Rayuela de Julio Cortázar (2006 [1963]). Las primeras narrativas hipertextuales que utilizan tecnología digital surgen en las décadas de 1980 y 1990. Algunos ejemplos destacados son Uncle Roger de Judy Malloy $\left(1995\right.$ [1986]) ${ }^{3}$ y Sunshine 69 de Bobby Rabyd (1996). Estas obras textuales son leídas mediante hipervínculos que permiten navegar distintos eventos de una historia en el orden en que los lectores decidan. Lo que estas obras ofrecen, sin embargo, son distintas formas de ordenar la secuencia de hechos, pero no necesariamente líneas de tiempo alternativas. Ejemplos de narrativas multilineales en medios típicamente lineales son la serie de libros Elige tu Propia Aventura y, más recientemente, el episodio Bandersnatch (Brooker \& Slade, 2018) de la serie Black Mirror.

El medio del videojuego difiere de los ejemplos arriba mencionados en que posee la capacidad de simular mundos ficcionales que cambian de estado de acuerdo con mecánicas predeterminadas. Estas mecánicas permiten inputs por parte de los jugadores, quienes pueden así producir eventos en el mundo ficcional del juego. Los cambios de estado producidos por los jugadores y por el juego (desde un pixel que titila hasta un avatar tridimensional en movimiento) permiten a los jugadores percibir el paso del tiempo en los videojuegos tal y como lo hacemos en la realidad-es decir, observando eventos en nuestro entorno (Wittmann, 2009, 2011). Los jugadores pueden a su vez controlar el paso del tiempo simulado de diferentes formas, de acuerdo con lo que el juego en cuestión permita: pueden pausarlo, acelerarlo, ralentizarlo, rebobinarlo y restablecer estados previos. Estas últimas dos propiedades de la temporalidad de un videojuego conducen a un tipo de multilinealidad que, por un lado, se diferencia de la novela de Ts'ui Pên por tratarse de simulaciones en lugar de texto, pero que, por otro lado, ofrece caminos y realidades alternativas en una escala más cercana a la descrita por Borges sobre la novela ficticia. 
El mundo simulado de un videojuego puede responder a inputs automáticamente y generar por si solo las líneas temporales alternativas, permitiendo así mayor granularidad en las variaciones y, por lo tanto, números drásticamente superiores de escenarios posibles. Si en un videojuego se puede elegir entre un camino que va hacia la izquierda y otro que va hacia la derecha, aquí ya hay dos posibles líneas en las que la historia puede desarrollarse. Pero también se puede elegir transitar el mismo camino de formas distintas: corriendo o caminando; deteniéndose a apreciar el paisaje o moviéndose con determinación y sin pausa; andando en línea recta o explorando cada recodo en el espacio. Estas decisiones alteran la relación de los jugadores con el personaje y con el espacio, otorgando distintos valores semánticos a la ficción.

En el caso de la literatura, el cine o la televisión, el contenido tiene que ser creado por uno o más autores, hecho que limita la cantidad de senderos que se pueden ofrecer en una historia. No obstante, la simulación posee limitaciones de otro tipo. Aún es imposible simular lenguaje en forma realista, por ejemplo. Por este motivo, si un videojuego incluye personajes que pueden conversar entre sí, los diálogos son concebidos por escritores y están sujetos a las mismas limitaciones de los medios tradicionales.

\section{Sobre Bucles y Marmotas}

Entre las narrativas tradicionales y unilineales, quizás la más citada por su similitud con el medio del videojuego sea la película El Día de la Marmota (Groundhog Day, Albert \& Ramis, 1993). ${ }^{5}$ En este film, el protagonista Phil Connors (interpretado por Bill Murray), se ve atrapado en un bucle temporal que lo fuerza a vivir la epónima jornada indefinidamente. La historia comienza cuando Phil llega al pueblo de Punxsutawney, en Pennsylvania, para cubrir la celebración por cuarto año consecutivo en su rol de reportero para un canal de televisión. Luego de realizar su trabajo renuentemente, una tormenta de nieve fuerza al protagonista y su equipo a volver a su posada y pasar una noche más en el pueblo. Phil despierta en lo que parece ser el día siguiente, pero descubre gradualmente que está reviviendo el día de la marmota, y él es el único que lo sabe.

Este loop se repite un número indeterminado de veces y produce una serie de reacciones en Phil Connors. En un primer momento, como es de esperar, su reacción es de desconcierto. Una vez que comprende lo que está sucediendo, comienza a desesperar y poner a prueba los límites de este extraño fenómeno, dado que las consecuencias de sus acciones se esfuman con la próxima iteración. En el paroxismo de su tormento, Phil intenta quitarse la vida repetidas veces, siempre con el mismo resultado: Con la misma certidumbre de que a la mañana siguiente va a salir el sol, Phil se despierta en la habitación de la posada en Punxsutawney en el día de la marmota.

Cuando Phil finalmente acepta su situación, comienza a percibirla de manera más positiva. Un personaje clave aquí es Rita (interpretada por Andie MacDowell), productora del canal de noticias. Phil sigue los consejos de su colega y comienza a comportarse de otro modo. Entre otras cosas, aprende a hablar francés, a tocar el piano y esculpir en hielo (hechos que insinúan que el ciclo se repite por un período extendido de tiempo). También ayuda a distintos habitantes del pueblo utilizando su capacidad para "ver el futuro". Es 
decir, al principio Phil interpreta sus circunstancias como un suplicio y luego como una fuente de oportunidades para aprender y asistir a otros. Los intentos del protagonista de seducir a Rita en su período de desesperación habían fracasado rotundamente, pero este renovado Phil despierta el interés de su colega y un romance comienza (repetidas veces debido al bucle temporal) entre ambos. El hechizo finalmente se rompe una vez que Phil realiza cambios definitivos en su carácter, reemplazando su modo egoísta y rencoroso con un carácter generoso y compasivo.

Phil Connors está atrapado en un laberinto temporal. Encontrar la salida de un laberinto de este tipo no consiste en caminar por una senda particular en el espacio, sino en producir los eventos que permiten que la flecha del tiempo pueda continuar hacia adelante de un modo prefijado-ya sea por una fuerza mayor o por nuestras propias aspiraciones. En el caso de Phil, estos eventos son los que llevan al perfeccionamiento de su carácter y su cambio de actitud ante otros. ${ }^{6}$

Los jugadores de videojuegos se ven involucrados con frecuencia en situaciones similares a la que narra El Día de la Marmota. Jugar un videojuego de un jugador implica, en gran medida, someterse a un proceso de prueba y error en el que se busca producir los eventos que permitirán avanzar hasta el final o conseguir el efecto deseado. Parte de este proceso consiste en volver atrás en el tiempo cargando partidas guardadas para intentar una porción del juego nuevamente (cf. Aarseth, 1999; Nitsche, 2007). Así y todo, cabe destacar algunas diferencias importantes entre la película en cuestión y el típico videojuego. Por un lado, a diferencia de Phil Connors, los personajes de videojuegos no suelen estar al tanto de las iteraciones. Son los jugadores quienes están en la posición del personaje de Bill Murray. Es decir, son los jugadores quienes son castigados con una repetición cuando pierden, pero a la vez tienen la posibilidad de volver atrás en el tiempo, borrando así sus errores y aprendiendo de ellos. Otra diferencia sustancial es que los jugadores normalmente ejercen cierto control sobre estas repeticiones. Mientras que Phil Connors no podía evitar comenzar el día desde el inicio, los jugadores pueden normalmente guardar el estado del juego con regularidad, para conservar así su progreso y evitar volver al principio cada vez que fracasan.

No obstante, el paralelo entre los videojuegos y El Día de la Marmota es claro. Esto me ha llevado previamente a utilizar el término Efecto del Día de la Marmota (EDM) ${ }^{7}$ para denominar al proceso de volver atrás en el tiempo de un juego con conocimiento sobre su futuro (Alvarez Igarzábal, 2016). Para que el EDM ocurra, se tienen que cumplir dos condiciones: (1) Los jugadores tienen que perder progreso y (2) el juego tiene que volver a un estado previo. Estos dos aspectos pueden parecen el mismo, pero perder progreso no siempre implica retroceder a un estado anterior. En el caso de juegos con permadeath (muerte permanente) y contenido generado proceduralmente, cuando el personaje muere, todo el progreso se pierde. Al ser la muerte del personaje irreversible, solo queda la opción de comenzar una partida nueva, creando un personaje nuevo. Pero el contenido generado proceduralmente no va a ser el mismo si se comienza de cero y, por lo tanto, esto implica jugar en un escenario distinto. Un ejemplo de este caso es el juego Rogue (Toy \& Wichman, 1980) y el género roguelike inspirado por el mismo. Igualmente, si cuando los jugadores pierden el juego no vuelve a un estado previo, sino que retoma desde el mismo momento en el que se perdió, el EDM no ocurre. En Space Invaders (Taito, 1976), por ejemplo, cuando el cañón controlado por los jugadores es destruido, el juego resta una vida de la partida y continúa manteniendo 
el resto del estado del juego intacto-siempre y cuando al jugador le sobre al menos una vida. También podría suceder, al menos en teoría, que el juego vuelva a un estado previo pero no borre el progreso realizado. Un ejemplo hipotético sería un flashback en el que se vuelve a un estado anterior del juego. El juego Horizon Zero Dawn (Guerilla Games, 2017) comienza en la infancia de la protagonista, Aloy. Luego de jugar una serie de misiones, Aloy crece y el juego continúa hasta el final con el personaje en su juventud temprana. Pero podría ser el caso que el juego siga ofreciendo esporádicamente misiones que vuelven al pasado, suministrando nueva información sobre la infancia de Aloy. En este caso, esos flashbacks volverían a un estado anterior del juego, pero los jugadores no perderían progreso. Una vez terminada la secuencia que vuelve al pasado de la protagonista, el juego volvería al presente, retomando su estado más avanzado.

Visualizar el EDM puede ayudar a comprender mejor su naturaleza y el impacto que tiene en juegos con narrativas. Para ello, me valdré de un modelo desarrollado por el investigador Jesper Juul.

\section{El EDM en el Modelo de Mapeo Temporal de Juul}

Para ilustrar el EDM, el modelo de mapeo temporal propuesto por Jesper Juul en su libro Half-Real: Video Games Between Real Rules and Fictional Worlds (2005) es de gran utilidad. En su modelo, Juul grafica dos líneas de tiempo: una para el tiempo de juego ("play time") y otra para el tiempo ficcional ("fictional time"). La primera línea (arriba en la figura 1) representa el tiempo real que transcurre al jugar y la segunda (abajo en la figura 1) representa el tiempo que pasa en el mundo ficcional del juego. La figura 1 también posee flechas verticales que apuntan hacia abajo entre las dos líneas de tiempo. Estas flechas representan la proyección ("projection"), el efecto que las acciones realizadas en el tiempo de juego tienen sobre el tiempo ficcional. Si presiono la tecla "W" en mi teclado, por ejemplo, esta acción se proyecta en el mundo ficcional cuando el personaje en el juego se mueve hacia adelante.

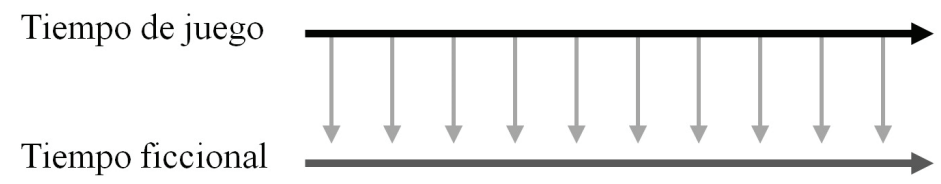

Figura 1. Modelo de mapeo temporal. Juul, J. (2005). Half-Real. Video Games between Real Rules and Fictional Worlds. Cambridge: MIT Press.

Juul agrega otros elementos a esta sencilla versión del modelo que grafican diferencias entre las dos líneas de tiempo, pero estos no son relevantes para el presente análisis. En cambio, voy a agregar algunos elementos propios para representar las características del EDM (cf. Alvarez Igarzábal, 2016). ${ }^{9}$ En la figura 2 se pueden observar cuadrados sobre la línea de tiempo de juego. Estos indican puntos en los que se guardó la partida. De estos 
cuadrados descienden líneas que señalan los puntos dónde la partida guardada coincide con el tiempo ficcional. La línea de tiempo ficcional está ahora dividida en segmentos de distinta magnitud ordenados en forma de escalera, de modo tal que se solapan en distinta medida. Estos segmentos terminan en una $\mathrm{X}$ que representa el momento en que se pierde la partida o por algún motivo se decide volver atrás en el tiempo. El tiempo ficcional cobra así un aspecto más aproximado al que tiene normalmente durante una partida: El juego comienza y se desarrolla hasta que el jugador pierde. El tiempo ficcional vuelve entonces al último estado guardado y retoma desde ahí. Este proceso se repite hasta que el jugador finaliza el juego o la partida.

Tiempo de juego

Tiempo ficcional

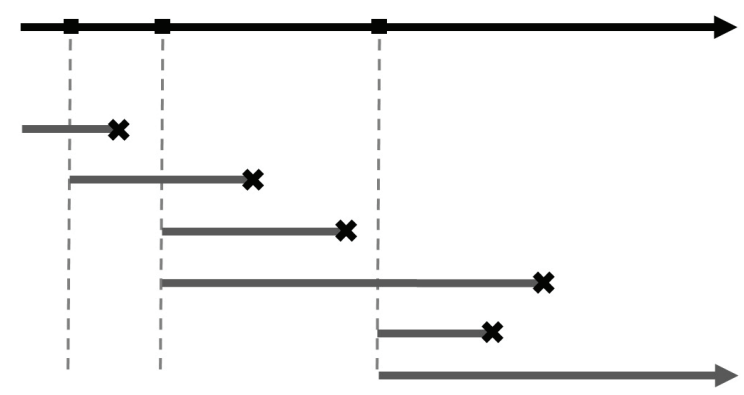

Figura 2. El EDM. Imagen de mi autoría basada en el modelo de Juul (2005)

Cada segmento de la línea de tiempo ficcional representa un camino tomado en el laberinto del tiempo que concluye en un evento equivalente a un callejón sin salida. El camino que lleva al personaje a la salida del laberinto es representado por la suma de aquellos cortes del tiempo ficcional que llevaron a la sucesión de partidas guardadas que a su vez condujeron al final del juego.

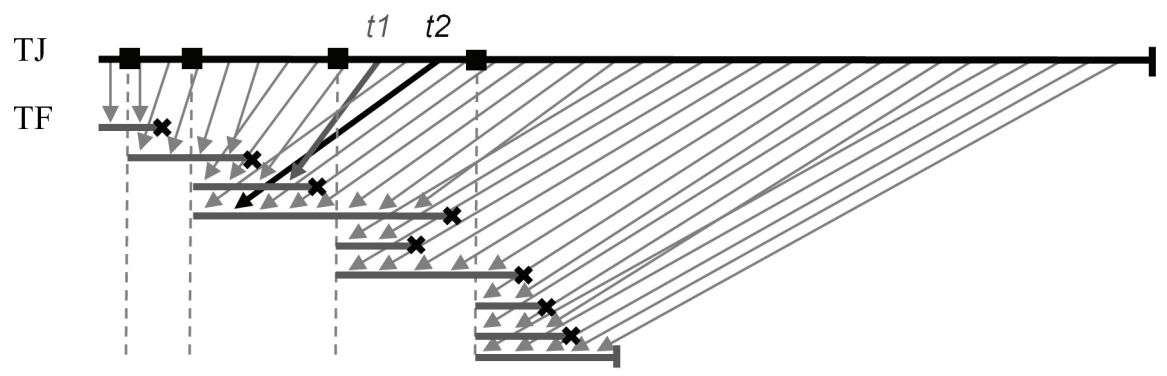

Figura 3. El EDM con la proyección del modelo de Juul (2005) 
Las líneas de tiempo en la figura 2 terminan en puntas de flecha, dado que el gráfico representa una porción de una partida hipotética que continúa. En la figura 3 se puede observar la representación de una partida completa (también hipotética) con el agregado de la proyección. Estas líneas de proyección, a diferencia del modelo original de Juul, abandonan su posición vertical, inclinándose hacia la izquierda en un ángulo cada vez más pronunciado a medida que se alejan del inicio de la línea de tiempo de juego. Esto sucede, claro, porque la línea de tiempo de juego prosigue su inexorable curso hacia adelante, mientras que la línea de tiempo ficcional zigzaguea, progresando más lentamente que la primera. En esta última figura hay dos líneas de proyección acentuadas con valores y grosores distintos al resto, rotuladas $\mathrm{t} 1$ y $\mathrm{t} 2$. T1 sucede primero en el tiempo de juego y t 2 más adelante, pero su proyección en el tiempo ficcional es inversa: $t 1$ ocurre después que $t 2$, solo que en un segmento superior. Este fenómeno ilustra el origen de uno de los dos problemas que resultan del EDM que voy a analizar a continuación.

\section{Problemas con el EDM}

El primer problema que se presenta a través del EDM es una paradoja temporal producida por la brecha entre el conocimiento de los jugadores y el personaje que estos controlan. Cuando un jugador vuelve atrás en el tiempo ficcional y juega una porción del juego nuevamente, este posee conocimiento sobre lo que va a suceder en el futuro (cf. Nitsche, 2007, p. 148). Es decir, si el personaje está por entrar a un espacio nuevo, el jugador ya sabe qué le espera ahí dentro (por ejemplo, cuántos enemigos y en dónde están ubicados). El personaje, en cambio, vuelve a un estado anterior junto con el resto del mundo ficcional, perdiendo así el conocimiento sobre futuros acontecimientos que se le puede atribuir. Como el personaje es controlado por alguien que posee este conocimiento, este se comporta de acuerdo a información que no tiene.

Esta paradoja no es necesariamente un problema. De hecho, en videojuegos en los que la historia es secundaria en relación a la mecánica y el perfeccionamiento de habilidades, esta consecuencia del EDM suele ser irrelevante. En juegos en los que el foco está en el desarrollo de una narrativa o de un personaje, en cambio, la paradoja puede presentar dificultades. ${ }^{10}$ Es por este motivo que en un juego como Heavy Rain (Quantic Dream, 2010), en el que la historia es el principal foco, no ofrece la opción de guardar y cargar partidas. Heavy Rain no tiene pantallas de game over ni condiciones que detienen el progreso del juego. Cada vez que los jugadores toman una decisión en la historia, el juego sigue adelante sin importar las consecuencias. Hagan lo que hagan los jugadores, el juego va a llegar a una conclusión, pero siempre influenciada por sus propias acciones. Heavy Rain es un laberinto temporal con distintas salidas, algunas satisfactorias y otras decepcionantes. Está en manos de los jugadores dirigir la historia hacia el final que ellos consideran deseable. David Cage, el diseñador y escritor de Heavy Rain, expresó la motivación detrás de la imposibilidad de guardar y cargar partidas en una entrevista con G4TV (Berghammer, 2009), antes del lanzamiento del juego. Cage afirmó que quisiera que los jugadores jueguen al juego solo una vez porque "that's life. Life you can only play once" (ibíd.). Y continuó: 
"I would like people to have this experience that way, but the game allows you play [sic!] as many times as you want, of course, and I'm fine with that, but the right way to enjoy Heavy Rain is really to make one thing because it's going to be your story. It's going to be unique to you. It's really the story you decided to write, and that will be a different story from someone else. And, again, I think playing it several times is also a way to kill the magic of it" (ibíd.).

Tres años más tarde, Cage repitió este argumento en una entrevista con Videogamer (Kelly, 2012), esta vez conversando sobre Beyond: Two Souls (Quantic Dream, 2013) y en 2016 lo hizo nuevamente en conversación con Gamespot (O’Dwyer, 2016) sobre Detroit: Become Human (Quantic Dream, 2018).

Cabe destacar que Heavy Rain (al igual que los juegos posteriores de Quantic Dream) posee un menú para seleccionar capítulos ya terminados, otorgando así la posibilidad de reiniciar partes de la historia-y los jugadores también tienen la opción de comenzar el juego de cero nuevamente. Es decir que el EDM puede tener lugar, pero la apuesta de Cage es que los jugadores se involucren con los personajes y la historia de modo tal que prefieran seguir adelante con el juego a deshacer decisiones y perder progreso reiniciando un capítulo, que no se juega tan rápido nuevamente. A su vez, no siempre está claro qué efectos tienen las decisiones tomadas, ni cuándo estos efectos van a tener lugar. Esta ambigüedad, sumada a la pérdida de progreso y la afirmación de Cage, demuestra una clara intención de desincentivar a los jugadores tentados a volver el tiempo atrás. Esto tiene sentido, considerando que el EDM puede generar distancia entre personajes y jugadores. El segundo problema se relaciona con las palabras de Cage y uno de los géneros históricamente más aclamados de la ficción: la tragedia. Consideremos la siguiente afirmación del diseñador de videojuegos Jesse Schell:

"[tragedy is] not really a thing for us. If we're doing an interactive Romeo and Juliet, what happens? Oh, my God, she died! Well, let me go back to the checkpoint, we're gonna fix that up (...) I'm not saying doing tragedy is impossible in video games. It's just hard" (GDC, 2013).

Los eventos en los videojuegos pierden su carácter definitivo como consecuencia del EDM, hecho que presenta evidentes dificultades a la hora de narrar una historia trágica. ${ }^{11} \mathrm{La}$ forma utilizada con mayor frecuencia hoy en día (al menos en títulos AAA) para sortear este obstáculo es el uso de cutscenes, es decir, clips de animación que interrumpen la partida para enfocarse en un aspecto puramente narrativo del juego-una alternativa (más popular en el pasado) es narrar la historia con texto. Luego de que la cutscene finaliza, el juego retoma su modo interactivo con las nuevas circunstancias demarcadas por la historia.

Como dijo el académico Henry Jenkins (2004, p. 8): “[t]he introduction needs to establish the character's goals or explain the basic conflict; the conclusion needs to show the successful completion of those goals or the final defeat of the antagonist". La introducción y la conclusión pueden ser entonces narrados en forma de cutscenes y lo que pasa entre medio puede estar sometido a cierto nivel de variación de acuerdo a las acciones que realizan 
los jugadores. Actualmente, un gran número de juegos incluyen clips de video de este modo, incluso entre medio de la acción. La cutscene final puede así convertir el triunfo de los jugadores sobre los obstáculos del juego en un desenlace negativo para el personaje, ofreciendo un final trágico.

John Marston, el protagonista del western Red Dead Redemption (Rockstar San Diego, 2010), encuentra un final de este tipo. Luego de colaborar con el Bureau of Investigations (el FBI de principios del siglo XX) en el ajusticiamiento los antiguos miembros de su banda, el otrora delincuente se retira a vivir una vida pacífica en una granja con su mujer e hijo. Pero los agentes traicionan su pacto con Marston e invaden su propiedad con la ayuda de soldados y oficiales. Esto desata un tiroteo que finaliza en un encuentro frontal entre el protagonista y más de una docena de oficiales. El evento se desarrolla, como es normal en este título, como una yuxtaposición de cutscenes de carácter puramente narrativo y pasajes interactivos en que los jugadores son invitados a utilizar mecánicas de shooter. Finalmente, Marston y su familia se esconden en el establo. Un clip narrativo muestra como madre e hijo escapan a caballo por la puerta trasera, mientras los oficiales esperan enfrente, fusiles y pistolas en alto. Marston decide quedarse atrás y terminar con el asunto. Abre la puerta frontal del establo e inmediatamente después el jugador tiene una última oportunidad de eliminar a sus enemigos. La superioridad numérica de los antagonistas es clara y, antes de que el jugador pueda despacharlos, una última cutscene concluye la confrontación. El jugador no tiene otra opción que ser espectador del desenlace, en el que Marston se convierte en el blanco de un torrente de proyectiles. En un giro final sorpresivo, el jugador se encuentra repentinamente controlando a Jack, el hijo, quien cabalga con su madre. Al escuchar los disparos, la mujer ordena a Jack (es decir, al jugador) volver a la granja para ayudar a Marston, pero solo encuentran su cuerpo sin vida.

Este ejemplo ilustra como los videojuegos pueden desembocar en un desenlace trágico restringiendo las opciones de los jugadores y encauzando el desarrollo de la acción mediante cortes no interactivos. El diseñador Chris Crawford—al igual que Schell—no está convencido de que este sea el método ideal para explotar el potencial narrativo del medio: "The story itself is noninteractive, and the game itself lacks dramatic content. You interact with the nonnarrative game, then see some non-interactive story, then interact some more with the game, then see more story, and if you alternate between the two fast enough, it becomes an 'interactive story'-right?" (Crawford, 2003, p. 260).

Este comentario sardónico no desmerece necesariamente el valor de un juego como Red Dead Redemption, que a todas luces fue un éxito crítico y comercial, ${ }^{12}$ pero sí subraya la relación conflictiva entre interacción y narrativa. A su vez, Crawford lamenta que los desarrolladores de videojuegos no se esmeren más en la búsqueda de formas de contar historias con el medio que no involucren interrupciones con relatos lineares ni sean más cercanas a películas interactivas-como es el caso de los juegos de Quantic Dream. ${ }^{13}$

Schell considera que el videojuego no se presta para la tragedia presuntamente no por ignorar la existencia de juegos como Red Dead Redemption (un título que difícilmente escaparía su atención), pero por reconocer las características del medio con el que trabaja. Al desarrollar una historia puramente interactiva, en teoría existe siempre la posibilidad 
de comenzarla nuevamente para deshacer un desenlace trágico-aunque se restrinjan las posibilidades de los jugadores para volver atrás en el tiempo rehuyendo checkpoints o la opción de guardar y cargar partidas.

\section{Conclusión}

El medio del videojuego es joven y el arte de la narración interactiva y multilineal está todavía en desarrollo. Por lo tanto, aún no hay respuestas claras a los desafíos que este tipo de ficciones traen consigo. La capacidad del videojuego para convertir a los jugadores en protagonistas de historias está a la vista. Al controlar un personaje, se vuelven responsables de sus decisiones y de las consecuencias las mismas. Esto que el videojuego realiza con facilidad es difícil de duplicar en otros medios, pero es a la vez su fortaleza y su punto débil. La libertad de los jugadores de tomar decisiones entra en conflicto con el control que los autores acostumbran a tener sobre su historia en medios típicamente lineales. Sumado a esto, la maleabilidad del tiempo en los videojuegos presenta un desafío a la hora de narrar historias cuyo impacto estriba en la irreversibilidad del mismo, como en el caso de la tragedia. Así y todo, la visión de Borges en El Jardín de Senderos que se Bifurcan se ha convertido con este nuevo medio en una realidad concreta que ya está dando resultados fascinantes, aunque quizás todavía imperfectos. Pareciera que los desarrolladores de videojuegos se encuentran en un laberinto temporal propio, cuya salida es la integración armónica de narrativa e interacción; el "Hamlet en la holocubierta" profetizado por Murray (1997) dos décadas atrás. Todavía no está claro siquiera si este laberinto tiene una salida y, si la tiene, exactamente qué camino lleva hacia ella. Pero eso es lo fascinante de un tiempo como el actual, en el que podemos ser testigos del desarrollo de un nuevo medio y, junto con él, la evolución del arte milenario de la narrativa.

\section{Notas}

1. En este texto me voy a referir a las narrativas en las cuales la historia puede seguir dos o más caminos alternativos como "multilineales", pero podría también denominarlas "no lineales", otro término utilizado en la literatura sobre el tema. Si bien ambos conceptos son utilizados, elijo el primero por considerarlo más apropiado, dado que una historia que puede desarrollarse de distintas formas presenta más de una línea narrativa, mientras que el término "no lineal" sugiere su ausencia.

2. Cabe destacar que hay casos de narrativas multilineales en medios tradicionales, a las cuales el texto hará alusión en la sección siguiente. Estas, sin embargo, son la excepción, mientras que en el videojuego la multilinealidad es más bien la regla.

3. Uncle Roger puede leerse aquí: https://people.well.com/user/jmalloy/uncleroger/partytop.html

4. Sunshine 69 puede leerse aquí: http://sunshine69.com/noflash.html 
5. Entre otros, Janet Murray (1997, pp. 37-36), Espen Aarseth (1999, p. 37) y Mark J.P. Wolf (2002, p. 80) señalaron las semejanzas entre El Día de la Marmota y el medio del videojuego. Yo realicé un análisis en base a esta analogía-en el que baso algunos pasajes de este texto-en mi artículo The Groundhog Day Effect. Iterations in Virtual Space (Alvarez Igarzábal, 2016).

6. La película no explicita por qué motivo Phil se encuentra en esa situación, ni que fuerza lo somete a revivir el mismo día una y otra vez. Dicha información es de cualquier modo irrelevante, dado que el bucle posee fundamentalmente un carácter metafórico.

7. Groundhog Day Effect (GDE) en el artículo original en inglés.

8. Juul también presentó este modelo en su artículo Introduction to Game Time / Time to Play. An Examination of Game Temporality (Juul, 2004).

9. Hitchens (2006) y Nitsche (2007) extendieron el modelo de Juul en forma similar para representar bucles temporales, pero sus modelos no son del todo adecuados para ilustrar el punto de este artículo.

10. En un artículo previo describo algunas posibles soluciones a la paradoja producida por el EDM (Alvarez Igarzábal, 2016).

11. Una posible excepción son juegos con muerte permanente, conocida como permadeath, y diseño procedural de niveles. Estos aspectos serán discutidos más adelante en el texto.

12. Una declaración del publisher de Red Dead Redemption, Take Two Interactive Software (2015), de 2015 afirma que despacharon 14 millones de copias del juego-lo que indica un éxito comercial. El juego tiene un puntaje de $95 \%$ de la crítica y de $90 \%$ de los usuarios en Metacritic (s.f.).

13. Crawford hace más que lamentar estas circunstancias. Desde hace muchas años trabaja en el desarrollo de Storytron (previamente denominado Erasmatron), un sistema para diseñar historias interactivas (Crawford, sin fecha).

\section{Lista de Referencias bibliográficas}

Aarseth, E.J. (1999). Aporia and Epiphany in Doom and The Speaking Clock. The Temporality of Ergodic Art. En M.L. Ryan (Ed.), Cyberspace Textuality. Computer Technology and Literary Theory (pp. 31-41). Bloomington, Indiana: Indiana University Press.

Albert, T., Ramis, H. (Productores), \& Ramis H. (Director). (1993). Groundhog Day [cinta cinematográfica]. Estados Unidos: Columbia Pictures.

Alvarez Igarzábal, F. (2016). The Groundhog Day Effect. Iterations in Virtual Space. En S. Höltgen \& J.C. van Treeck (Eds.), Time to Play. Zeit und Computerspiel (pp. 225-246). Glückstadt: Verlag Werner Hülsbusch.

Berghammer, B. (2009). Changing the Game. The Quantic Dream Heavy Rain Interview Part Two. G4TV. Recuperado de http://www.g4tv.com/games/ps3/36147/heavy-rain/articles/68230/Changing-The-Game-The-Quantic-Dream-Heavy-Rain-Interview-Part-Two/

Borges, J.L. (2013 [1941]). Jorge Luis Borges. Cuentos Completos. Barcelona: Penguin Random House. 
Brooker, C. (Writer), \& Slade, D. (Director). (2018). Black Mirror: Bandersnatch [episodio de serie de televisión]. En McLean (Executive Producer), Black Mirror, Reino Unido: Netflix. Bush, V. (2003 [1945]). As We May Think. En N. Wardrip-Fruin \& N. Montfort (Eds.), The New Media Reader (pp. 35-47). Cambridge: MIT Press.

Cortázar, J. (2006 [1963]). Rayuela. Madrid: Punto de Lectura.

Crawford, C. (2003). Interactive Storytelling. En M.J.P. Wolf \& B. Perron (Eds.), The Video Game Theory Reader (pp. 259-273). New York: Routledge.

(s.f.). Storytron. Recuperado de https://www.storytron.com/

Engelbart, D. (2003 [1962]). Augmenting Human Intellect. A Conceptual Framework. En N. Wardrip-Fruin \& N. Montfort (Eds.), The New Media Reader (pp. 95-108). Cambridge: MIT Press.

GDC. (2013, Marzo). The Future of Storytelling: How Medium Shapes Story [video]. Recuperado de https://www.youtube.com/watch?v=BjrO-di22v8

Guerilla Games. (2017). Horizon Zero Dawn [videojuego]. Países Bajos: Sony Interactive Entertainment.

Hitchens, M. (2006). Time and Computer Games or "No, That's not What Happened". IE '06 Proceedings of the 3rd Australasian Conference on Interactive entertainment, 44-51. Recuperado de https://dl.acm.org/citation.cfm?id=1231902

Jenkins, H. (2004). Game Design as Narrative Architecture. En N. Wardrip-Fruin \& P. Harrigan (Eds.), First Person. New Media as Story, Performance, and Game (pp. 118-130). Cambridge: MIT Press.

Juul, J. (2004). Introduction to Game Time / Time to Play. An Examination of Game Temporality. En N. Wardrip-Fruin \& P. Harrigan (Eds.), First Person. New Media as Story, Performance, and Game (pp. 131-142). Cambridge, Massachusetts: MIT Press, 2004.

Juul, J. (2005). Half-Real. Video Games between Real Rules and Fictional Worlds. Cambridge: MIT Press.

Kelly, N. (2012). David Cage: “I remember how scared we were”. VideoGamer. Recuperado de: http://www.videogamer.com/ps3/heavy_rain/news/david_cage_i_remember_how_ scared_we_were.html

Licklider, J.C.R. (2003 [1960]). Man-Computer Symbiosis. En N. Wardrip-Fruin \& N. Montfort (Eds.), The New Media Reader (pp. 73-82). Cambridge: MIT Press.

Malloy, J. (1995 [1986]). Uncle Roger. Recuperado de https://people.well.com/user/jmalloy/ uncleroger/partytop.html

Metacritic. (s.f.). Red Dead Redemption. Recuperado de https://www.metacritic.com/game/ xbox-360/red-dead-redemption

Montfort, N. (2003). Introduction. The Garden of Forking Paths. En N. Wardrip-Fruin \& N. Montfort (Eds.), The New Media Reader (pp. 29-30). Cambridge: MIT Press.

Murray, J. (2003). Inventing the Medium. En N. Wardrip-Fruin \& N. Montfort (Eds.), The New Media Reader (pp. 3-11). Cambridge: MIT Press.

Murray, J. (1997). Hamlet on the Holodeck. The Future of Narrative in Cyberspace. Cambridge: MIT Press.

Nabokov, V. (2003 [1962]). Pálido Fuego. Barcelona: Anagrama. 
Nelson, T. (2003 [1965]). A File Structure for the Complex, the Changing, and the Indeterminate. En N. Wardrip-Fruin \& N. Montfort (Eds.), The New Media Reader (pp. 134-145). Cambridge: MIT Press.

Nintendo. (1985). Super Mario Bros. Japón: Nintendo.

Nitsche, M. (2007). Mapping Time in Video Games. Situated Play. Proceedings of DiGRA 2007 Conference, 145-151. Recuperado de http://homes.lmc.gatech.edu/ nitsche/ download/Nitsche_DiGRA_07.pdf

O’Dwyer, D. (2016). David Cage Interview at the E3 2016 Stage Show. Gamespot. Recuperado de https://www.gamespot.com/videos/david-cage-interview-at-the-e3-2016-stageshow/2300-6432977/

Quantic Dream. (2010). Heavy Rain [videojuego]. Francia: Sony Computer Entertainment.

Quantic Dream. (2013). Beyond: Two Souls [videojuego]. Francia: Sony Computer Entertainment.

Quantic Dream. (2018). Detroit: Become Human [videojuego]. Francia: Sony Interactive Entertainment.

Rabyd, B. (1996). Sunshine 69. Recuperado de http://sunshine69.com/noflash.html

Rockstar San Diego. (2010). Red Dead Redemption [videojuego]. Estados Unidos: Rockstar Games.

Taito. (1976). Space Invaders [videojuego]. Japón: Taito.

Take Two Interactive. (2015, Agosto 18). SEC Filings. Recuperado de http://ir.take2games. com/phoenix.zhtml? $c=86428 \& p=$ irol-SECText $\&$ TEXT $=$ aHR0cDovL2FwaS50ZW5rd2l 6YXJkLmNvbS9maWxpbmcueG1sP2lwYWdlPTEwNDQ0MDQ2JkRTRVE9MCZTRVE9MCZTUURFU0M9U0VDVElPT19FTlRJUkUmc3Vic2lkPTU3

Toy, M. \& Wichman, G. (1980). Rogue [videojuego]. Estados Unidos: Epyx.

Wittmann, M. (2009). The Inner Experience of Time. Philosophical Transactions of The Royal Society 364, 1955-1967. https://doi.org/10.1098/rstb.2009.0003

Wittmann, M. (2011). Moments in Time. Frontiers in Integrative Neuroscience 5, article 66. https://doi.org/10.3389/fnint.2011.00066

Wolf, M.J.P. (2002). Time in the Video Game. En M.J.P. Wolf(Ed.), The Medium of the Video Game (pp. 77-91). Austin: University of Texas Press.

\begin{abstract}
Video games grant players the capacity to save and load game states, allowing them to travel back in game time. Whenever an unwanted event takes place, players can load a previously saved state and replay a portion of the game to achieve the desired results. Two challenges arise from this mechanic when telling stories: 1) The capacity to undo events is incompatible with tragedy and 2) a temporal paradox emerges: When players travel back in time, they have knowledge about the future. The player character cannot possess this knowledge, since it was reset with the rest of the fictional world. Nevertheless, it behaves as if it possessed it, given that it is controlled by the player.
\end{abstract}

Key words: video games - narrative - multilinear narrative - fiction - interaction - time literature. 
Resumo: Os videogames oferecem a opção de salvar e carregar checkpoints, permitindo aos jogadores retroceder no tempo do jogo. Cada vez que um evento indesejado ocorre, eles podem carregar um estado anterior e jogar uma nova partida, a fim de alcançar o resultado desejado. Dois desafios surgem dessa mecânica ao contar histórias: 1) A capacidade de desfazer eventos é incompatível com a tragédia e 2) surge um paradoxo temporal: ao retornar no tempo, o jogador passa a conhecer o futuro. No entanto, o personagem que ele controla não deveria possuir esse conhecimento, já que ele mesmo é reconstituído junto com o restante do mundo ficcional. Ainda assim, uma vez que é controlado pelo jogador, se comporta como se o tivesse.

Palavras-chave: videogames - narrativa - narrativa multilinear - ficção - interação - tempo - literatura.

[Las traducciones de los abstracts fueron supervisadas por el autor de cada artículo] 